\title{
Pond fractals in a tidal flat
}

\author{
B. B. Cael" and Bennett Lambert \\ Massachusetts Institute of Technology, 77 Massachusetts Avenue, Cambridge, Massachusetts 02139, USA \\ and Woods Hole Oceanographic Institution, 86 Water Street, Woods Hole, Massachusetts 02543, USA \\ Kelsey Bisson \\ Earth Research Institute, University of California, Santa Barbara, California 93106, USA
}

(Received 25 August 2015; published 19 November 2015)

\begin{abstract}
Studies over the past decade have reported power-law distributions for the areas of terrestrial lakes and Arctic melt ponds, as well as fractal relationships between their areas and coastlines. Here we report similar fractal structure of ponds in a tidal flat, thereby extending the spatial and temporal scales on which such phenomena have been observed in geophysical systems. Images taken during low tide of a tidal flat in Damariscotta, Maine, reveal a well-resolved power-law distribution of pond sizes over three orders of magnitude with a consistent fractal area-perimeter relationship. The data are consistent with the predictions of percolation theory for unscreened perimeters and scale-free cluster size distributions and are robust to alterations of the image processing procedure. The small spatial and temporal scales of these data suggest this easily observable system may serve as a useful model for investigating the evolution of pond geometries, while emphasizing the generality of fractal behavior in geophysical surfaces.
\end{abstract}

\section{INTRODUCTION}

Power-law distributions are characteristic of scale-free phenomena and occur widely in nature [1]. In particular, a hallmark of the study of self-similar surfaces (e.g., continuum percolation theory and statistical topography) is the power-law distribution of areas enclosed by contours of level surfaces. This is widely observed in geophysical systems through both connected and closed bodies of water [2-4]. Such coastlines typically have a "rough" texture, characteristic of random fractals $[5,6]$.

In the past decade, celebrated papers have demonstrated the existence of such power-law-distributed, fractal water bodies in geophysical systems [2,3,7-9]. Importantly, these studies established a link between power-law-distributed terrestrial lakes and fractal behavior over large dynamic ranges. Research on Arctic melt ponds revealed a fractal dimension transition from isolated simple ponds to larger, self-similar interconnected ponds, characteristic of a monoscale topographic relief $[9,10]$. Indeed, in recent work the level sets of random surfaces constructed from sea ice topography data yield the observed transition in fractal dimension, thus suggesting the advantage of such methods in studying geophysical surfaces [11]. It is well understood that lakes and melt ponds are climatically influential for their roles in the carbon cycle [12-14] and icealbedo feedbacks [15-18], respectively. Often their locations and temporal scales (seasonal in the melt pond case, often far longer in the lake case) of their evolution make them hard to observe and quantify robustly $[19,20]$. Characterizing

\footnotetext{
*bcaelb@mit.edu
}

Published by the American Physical Society under the terms of the Creative Commons Attribution 3.0 License. Further distribution of this work must maintain attribution to the author(s) and the published article's title, journal citation, and DOI. their spatiotemporal distributions makes their impact easier in principle to quantify because their total coverage can be calculated knowing just the slope of their distribution and the number of elements at a particular size.

Herein, we extend the observed spatial and temporal scales of this geophysical fractal phenomenon to the ranges of centimeters and minutes. Data collected from a tidal flat at low tide in Damariscotta, Maine, show a power-law distribution of ponds over several orders of magnitude, from 1 to $1000 \mathrm{~cm}^{2}$, with a fractal dimension consistent with the predictions of percolation theory (see Ref. [21]) and a reasonable correlation length scale [9]. These statistical quantities are robust to alterations of the image processing technique and reveal a system statistically similar to lakes and melt ponds.

\section{DATA COLLECTION AND ANALYSIS}

Coastal Maine experiences pronounced tidal cycles, frequently generating mudflats in its intertidal zones. In particular, Days Cove $\left(44.03^{\circ} \mathrm{N}, 69.53^{\circ} \mathrm{W}\right)$ is a highly dynamic, low-gradient inlet adjacent to the Damariscotta River Estuary. While completely submerged at high tide, at low tide it exhibits channelized flow, and the rigid, slow-draining clay-sized mud of the flat promotes the ubiquitous formation of small ponds on the surface of the flat [see Figs. 1(a) and 1(b)]. These ponds are characterized by their rough edges and apparent scale-free behavior over a large dynamic range.

Low tide occurred at 11:45 a.m. on 25 July 2015, at which point the authors took images over pond ensembles. Images were taken with a suspended iPhone 6 using a 5- or 10 -s time lapse, at 1080-pixel resolution, from $4.5 \pm 0.5 \mathrm{~m}$ height, resulting in a field of view of approximately $3.3 \times$ $2.5 \mathrm{~m}^{2}$. Images included $8.25-\mathrm{cm}$-diam reference disks by which the images were rescaled, and were found to have a pixel resolution of $0.26 \pm 0.03 \mathrm{~cm}$; images contained $O\left(10^{3}\right)$ ponds, a sufficient quantity to be considered an ensemble. Uniformly overcast skies at the time of sample greatly minimized specular 


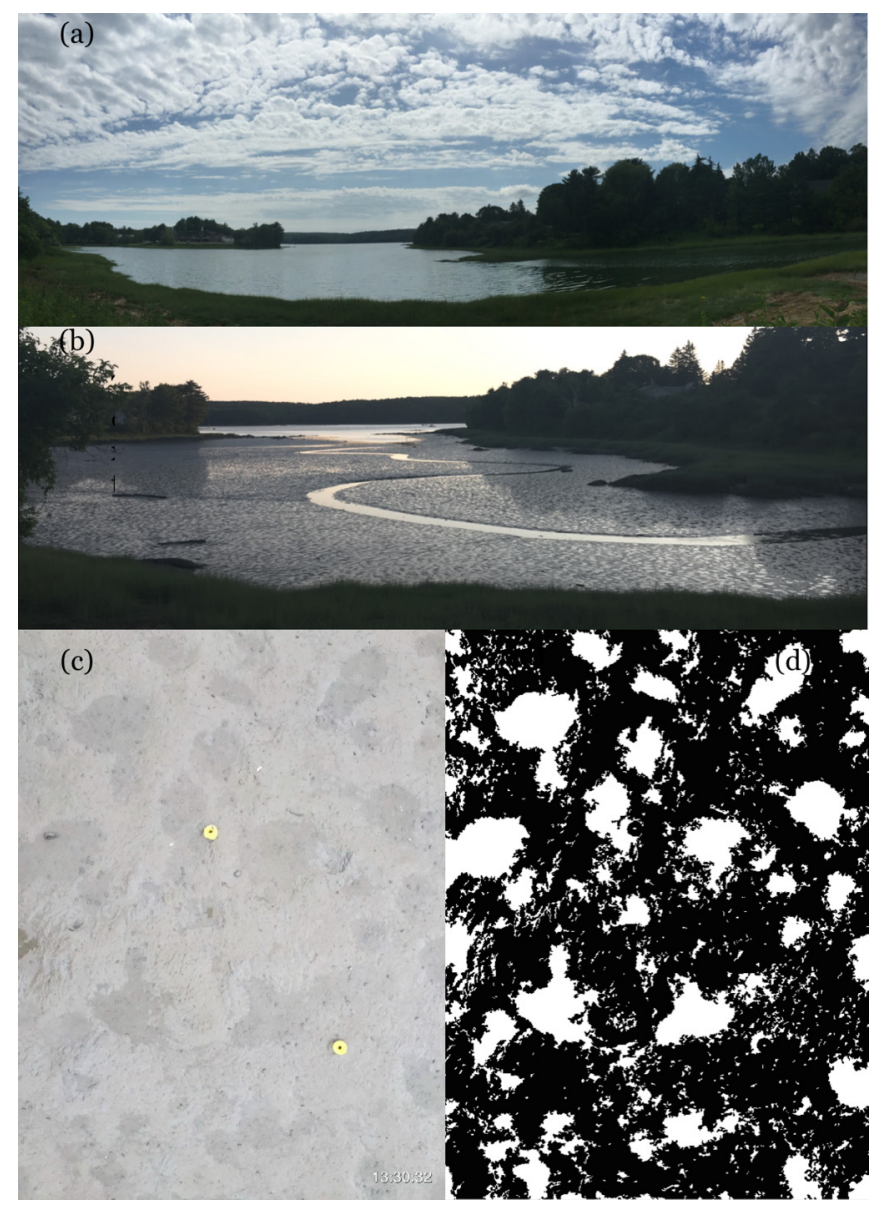

FIG. 1. (Color online) (a) Field site at Days Cove during high tide, (b) low tide later that day, with ponds appearing, (c) unprocessed overhead image, $3.3 \times 2.5 \mathrm{~m}^{2}$, with reference disk, and (d) processed black (mud) and white (pond) image detecting pond coverage.

glint, permitting optimal image acquisition. Only a subset of the tidal flat was sampled, due to the incoming tide, and ponds below 10 pixels were ignored to minimize the effect of measurement noise on the small tail of the samples. The distribution of ponds showed no significant variation in any measured statistic within the sampled areas, suggesting the imaged subset appropriately characterizes the flat and the absence of any large-scale gradients in the pond distribution. Individual pond volume varied negligibly over the sampling period, constraining the influence of seepage.

Images were processed using the MATLAB imaging toolbox; pond areas and perimeters were found using built-in MATLAB functions. Images were quality controlled manually to minimize double sampling and potentially problematic objects such as footprints; the final data set comprises 123 images, resulting in $O\left(10^{5}\right)$ ponds (precisely $n=130751$ ) which are generally well captured by the image processing [see Figs. 1(c) and 1(d)]. A series of tests was run to ascertain the role that confounding variables played in image analysis (e.g., edge effects, filtering, or minimum pond size considered). Adaptive histogramming was employed to improve detection of pond contours. Edge effects were not found to be influential; ignoring ponds that intersected the edge of the images had a negligible effect on area-perimeter relationships but revealed an expected bias against large ponds. These suggest sufficient pond diversity is captured within a given image. Mirroring images and tiling them to minimize edge effects yielded negligible effects on the statistics as well; across several image processing strategies all statistics were insensitive to processing details, with derived quantities changing only up to a few percent.

\section{RESULTS}

Following processing, the derived individual pond areas (a) were logarithmically binned and plotted as a cumulative distribution, or "rank-frequency function" $(f)[1]$ :

$$
f(a)=\int_{a}^{\infty} p\left(a^{\prime}\right) d a^{\prime},
$$

which reveals a robust power law over three orders of magnitude (from 1 to $1000 \mathrm{~cm}^{2}$ ) with a slope of $-0.67( \pm 0.04)$, indicating a power-law distribution with a slope $(\alpha)$ of $-1.67( \pm 0.04)$. The data were fit for both a power law over the $1-1000 \mathrm{~cm}^{2}$ range cited above, distributed as

$$
p(a) \sim a^{\alpha},
$$

as well as fit for a power-law distribution with a correlation length scale $\xi$ [9], the maximum radius above which ponds are exponentially scarce, distributed as

$$
p(a) \sim a^{\alpha} e^{-a /\left(\pi \xi^{2}\right)} .
$$

Uncertainty ranges of \pm 0.04 were taken from the total range across both fits and all image processing strategies. Figure 2 shows the rank-frequency function plotted with both distributions; $\pi \xi^{2}$ is approximately $2.0 \mathrm{~m}^{2}$, or $\xi \approx 0.80 \mathrm{~m}$. This correlation length scale is consistent with observations; very few ponds were observed at $O(1 \mathrm{~m})$ radial lengths. The correlation length scale is also well separated from the individual image sizes of approximately $3.3 \times 2.5 \mathrm{~m}^{2}$, suggesting that the images are of significant size to capture the pond statistics.

Fractal dimension $d_{f}$, defined by the perimeter-area $(\ell-a)$ relationship

$$
\ell \sim a^{d_{f} / 2}
$$

was computed by scattering ponds in perimeter-area space $[9,10,21]$; theoretically if the edges of the ponds are considered as unscreened hulls for continuum percolation clusters, i.e., the continuous lines which envelop the cluster, their fractal dimension would be predicted to be 4/3 [9]. As seen in Fig. 3, the slope of $2 / 3$ matches well with the scattered data. The match between data and the unscreened hull prediction suggests that the trapped water tends to erode all small features protruding above the water surface within ponds, consistent with the images upon inspection. A more rigid surface material might possibly retain small "islands" within the interior of ponds, thus increasing perimeter and decreasing area and so increasing fractal dimension; the softness of the mud in this flat causes the trapped water to erode these island features, thus making the unscreened hull ansatz a reasonable one.

Hohnegger et al. [10] observed a transition in fractal dimension of Arctic melt ponds with increasing pond area, 


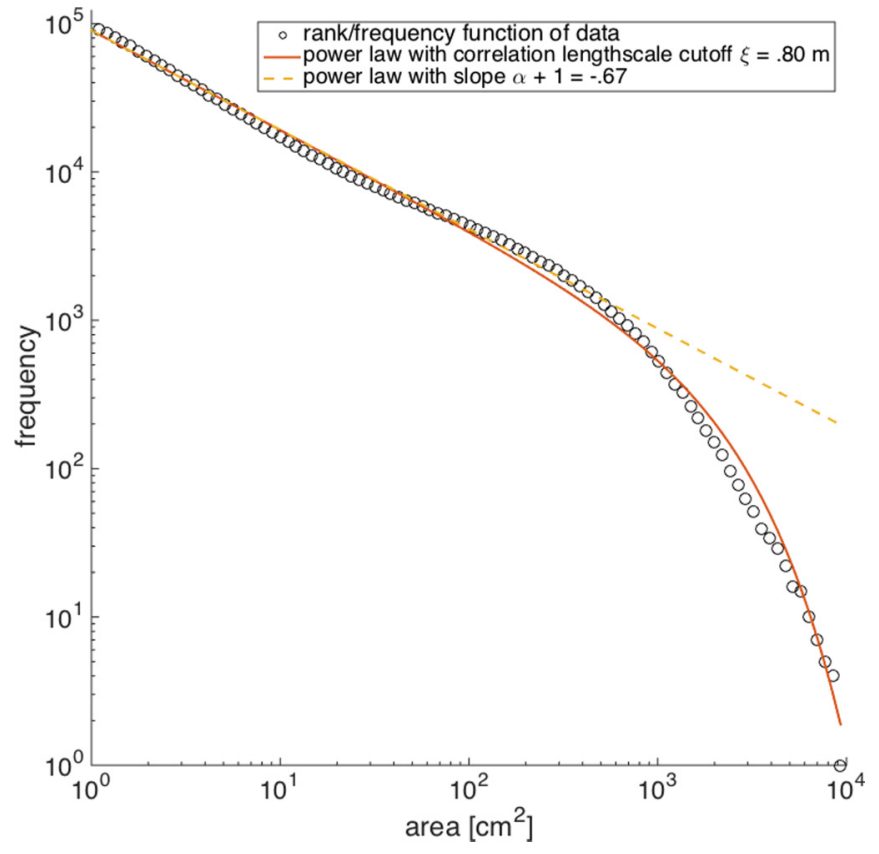

FIG. 2. (Color online) Rank-frequency function $(f)$ for pond areas. $f$ will have slope 1 greater than probability density function (PDF) due to being an integral; $f$ power-law fit has slope of $\alpha+1 \approx$ -0.67 (dotted yellow line), thus PDF has slope of $\alpha \approx-1.67 . f$ fit with correlation length scale $\xi \approx 0.80 \mathrm{~m}$ (solid red line), which results in an exponential tailing for large ponds. $r^{2}$ for both are $>0.99$ over their fitting domain.

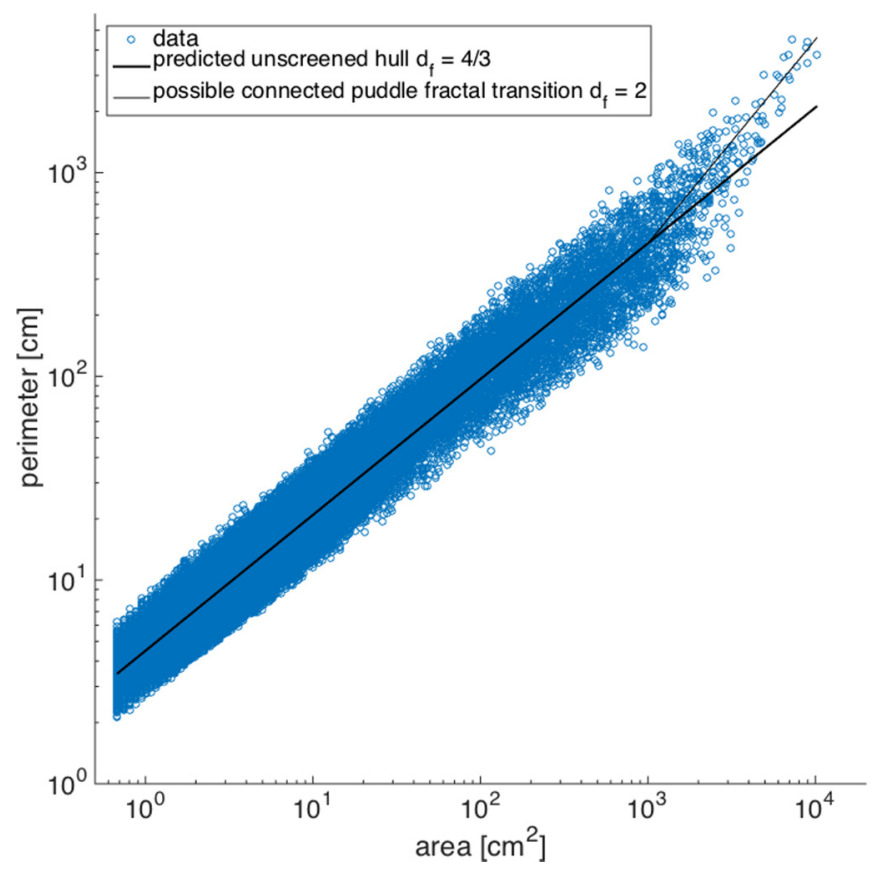

FIG. 3. (Color online) Area-perimeter scatterplot for pond fractal dimension. The thick line is the predicted slope of $4 / 3$ from percolation theory for unscreened hulls, with an $r^{2}>0.85$. The thinner line is representative of a possible transition towards connected ponds with fractal dimension $d_{f} \approx 2$ in the upper limit of pond sizes, which may not show up distinctly in this data set due to masking by the correlation length scale.
TABLE I. Summary of major findings. ${ }^{a}$

\begin{tabular}{lcc}
\hline \hline Pond fraction & $\varphi$ & 0.28 \\
PDF slope & $\alpha$ & -1.67 \\
Correlation length scale & $\xi$ & $0.80 \mathrm{~m}$ \\
Fractal dimension & $d_{f}$ & $4 / 3$ \\
\hline \hline
\end{tabular}

${ }^{a} r^{2}$ for power-law slope $>0.99 ; r^{2}$ for fractal dimension $>0.85$.

as individual ponds are actually composed of connected sets of smaller ponds. Towards the larger end of the ponds, the data reported here may possibly show a fractal transition as well; these data, however, remain inconclusive, and the larger connected ponds may be exponentially unlikely due to the correlation length scale. Between a single linear fit and a two-line piecewise linear fit intended to capture this transition quantitatively, the ratio of sum of squared errors of prediction between the two cases was $6553 / 6584 \approx 0.995$, yielding no shift in the adjusted $r^{2}$; this suggests that though visual inspection of the scattered data may suggest such a transition, it is statistically insignificant due to the rarity of large ponds in this data set.

The pond fraction of total sampled area is $\varphi \approx 28 \%$, suspected to be below the criticality threshold for most two-dimensional percolation systems (usually closer to $1 / 2$ ), consistent with the existence of a correlation length scale (which diverges at the percolation transition). See Table I for a summary or major findings.

\section{DISCUSSION}

The results strongly suggest a fractal pond pattern in tidal flats similar to those found in terrestrial lakes and Arctic melt ponds, albeit on different spatiotemporal scales and driven by different physical processes. While additional data are needed to determine what mechanisms drive the statistics of this process, e.g. the correlation length scale, the data do suggest the height of the mud is randomly distributed. The power-law distribution is robust over several orders of magnitude, as is the fractal area-perimeter relationship, and the deviation from a power law at large pond sizes is well fit by a correlation length scale effect, which is consistent with visual inspection of the ponds and smaller than the size of the collected images. These findings highlight the generality of the descriptions of continuum percolation or statistical topography for geophysical systems and may greatly enhance the ease with which such processes can be observed, due to the accessibility of the site, the spatial and temporal scales at which this phenomenon occurs here, and the affordability with which it can be measured as a result. The possibility of a connected fractal dimension transition exists at the tail of the measured distribution, though its existence is inconclusive based on this data set. Statistical information is robust to alterations of the image processing technique.

Small fluctuations in the rank-frequency function are found in all strategies of image processing and are visible in Fig. 2; these could be explained by noise in the data set due to a finite sample size, to second-order physical processes (e.g., erosion feedback of the ponds on the surface), to edge effects not solvable by the techniques employed herein, or to other 
unknown features of the system. The multiplicity of different species (including mussels; oysters; horseshoe, hermit, and other crabs; anemones; and, most prominently, small snails) in the tidal flat may have some impact on the pond evolution, as in Refs. [22,23]. While this type of pond distribution may not be expected to hold in all intertidal flats, e.g., those with steep slopes, it is reasonable to expect it may exist in similarly classified mudflats which present conditions that could support such pond structures, i.e., those with low slope, clayish mud, and large tidal range [24,25].

Our results invite the potential for time series work to understand pond evolution and its variations as a function of the advancing tide (water volume). Employing a quadcopter, for example, will generate larger field of view images whose analysis could be used to investigate the effects of increasing pond coverage, e.g., via an advancing tide, on these processes. Nonlinear numerical modeling of the feedbacks at the mudpond interface may also yield useful insights into the generality of this system's dynamics for future application elsewhere.

\section{ACKNOWLEDGMENTS}

This material is based upon work supported by the National Science Foundation Graduate Research Fellowship Program under Grant No. 2388357, the Gordon and Betty Moore Foundation, and the National Science Foundation, Award No. OCE-1315201.
[1] M. E. J. Newman, Contemp. Phys. 46, 323 (2005).

[2] J. A. Downing, Y. T. Prairie, J. J. Cole, C. M. Duarte, L. J. Tranvik, R. G. Striegl, W. H. McDowell, P. Kortelainen, N. F. Caraco, J. M. Melack, and J. J. Middleburg, Limnol. Oceanogr. 51, 2388 (2006).

[3] D. K. Perovich, W. B. Tucker III, and K. A. Ligett, J. Geophys. Res. 107, 8048 (2002).

[4] D. G. Tarboton, R. L. Bras, and I. Rodriguez-Iturbe, Water Resour. Res. 24, 1317 (1988).

[5] B. B. Mandelbrot, The Fractal Geometry of Nature (W. H. Freeman, New York, 1983).

[6] J. Kondev and C. L. Henley, Phys. Rev. Lett. 74, 4580 (1995).

[7] D.-J. Kim, B. Hwang, K. H. Chung, S. H. Lee, H.-S. Jung, and W. M. Moon, Proc. IEEE 101, 748 (2013).

[8] D. A. Seekell, M. L. Pace, L. J. Tranvik, and C. Verpoorter, Geophys. Res. Lett. 40, 517 (2013).

[9] M. B. Isichenko, Rev. Mod. Phys. 64, 961 (1992).

[10] C. Hohenegger, B. Alali, K. R. Steffen, D. K. Perovich, and K. M. Golden, The Cryosphere 6, 1157 (2012).

[11] B. Bowen, C. Strong, and K. M. Golden, J. Fractal Geom. (to be published).
[12] S. Sobek, G. Algesten, A.-K. Bergstrm, M. Jansson, and L. J. Tranvik, Global Change Biol. 9, 630 (2003).

[13] J. J. Cole and N. F. Caraco, Mar. Freshwater Res. 52, 101 (2001).

[14] J. A. Downing, Limnetica 29, 9 (2010).

[15] J. Hanesiak, J. J. Yackel, and D. G. Barber, Can. J. Remote Sens. 27, 433 (2014).

[16] D. K. Perovich, J. A. Richter-Menge, K. F. Jones, and B. Light, Geophys. Res. Lett. 35, L11501 (2008).

[17] D. K. Perovich, T. C. Grenfell, B. Light, and P. V. Hobbs, J. Geophys. Res. 107, 8044 (2002).

[18] J. Curry, J. Schramm, and E. Ebert, J. Climate 8, 240 (1995).

[19] B. Lehner and P. Döll, J. Hydrol. 296, 1 (2004).

[20] D. Alsdorf, D. Lettenmaier, and C. Vrsmarty, Eos 84, 269 (2003).

[21] A. J. Aharony, Stat. Phys. 34, 931 (1984).

[22] A. Mahadevan and L. Mahadevan, Engineering 1, 1 (2010).

[23] A. Mahadevan (private communication).

[24] K. R. Dyer, M. C. Christie, and E. W. Wright, Cont. Shelf Res. 20, 1039 (2000).

[25] K. R. Dyer, Geol. Soc. Spec. Publ. 139, 11 (1998). 